\title{
ON THE BOTTOM SHEAR STRESS DURING LONG WAVE RUNUP AND BACKWASH
}

\author{
Takenori Shimozono $^{1}$, Akio Okayasu ${ }^{1}$ and Teppei Mishima ${ }^{1}$
}

\begin{abstract}
Laboratory experiments were performed to examine flow characteristics during runup and backwash of a breaking solitary wave. Boundary layer flows were measured by LDV at two locations landward of the still shoreline under different bed conditions. Temporal variations of the bottom shear stress were estimated from nearbed velocity profiles with different methods. The shear stress determined through the logarithmic fitting roughly agreed with that deduced from a depth-integrated momentum balance during turbulent flow phases. The friction factors during the active phases of the runup and backwash were similar in magnitude except in the leading tip where the value sharply increased. A movable bed experiment was also carried out under the same geometry to discuss relations between the bottom shear stress and sediment transport. The result indicated clear dependencies of bed deformations on the magnitude and duration of the exerted shear stress.
\end{abstract}

Keywords: bottom shear stress; swash zone; wave runup; sediment transport; solitary wave

\section{INTRODUCTION}

Hydrodynamics and sediment transport in the swash zone are remaining areas of study in coastal engineering. Recent developments in computational models enable us to simulate wave fields and resulting sediment transport with the inclusion of the swash zone that is intermittently submerged by waves running up and down. In the swash zone, a loss of momentum due to the bottom shear stress is relatively important as large velocities are produced within a thin layer of water. Also in sediment transport simulations, the bottom shear stress plays a key role relating a flow state with sediment entrainment into water. Empirical formulae derived in steady flows are conventionally used in the models to estimate the bottom shear stress in spite of its highly transient property. For the model enhancement, it is essential to accurately evaluate the bottom shear stress from flow variables in consideration of the flow unsteadiness.

However, the flow characteristics in the swash zone are not well understood quantitatively. This is largely attributed to difficulties in flow measurements since the water is very thin and occasionally absent. Even so, a number of experimental investigations have been done to quantify the bottom shear stress both in laboratories and actual fields in the past decade. A common approach is to measure the flow velocity in the boundary layer and estimate the stress by fitting the velocity profiles to the logarithmic law. The different instruments such as LDV (Cox et al. 2001), ADVP (Archetti and Brocchini 2002) and PIV (Cowen et al. 2003) are employed in laboratory measurements of nearbed velocity profiles. The same approach is also applied to field investigations (Puleo and Holland 2002, Raubenheimer et al. 2004). Major problems of the approach are a difficulty in acquiring the velocity samples in leading and trailing edges and an uncertainty associated with the use of the logarithmic profile. More recently, direct measurements of the shear stress have been attempted to overcome the shortcomings of the indirect measurements (Barnes et al. 2007).

The main focus of the above studies was temporal variations of the bottom shear stress over the swash cycle. Especially, a difference in magnitude of the bottom shear stress between the runup and backwash was discussed in relation with turbulence characteristics. The most results suggested that a typical value of the friction factor is different between the runup and backwash implying the quadratic law with a constant factor produces a significant error in the model. However, the reported values of the friction factor are significantly different among the authors. There is still a large amount of uncertainty in the quantitative characteristics on the bottom friction under the swash motion. More investigations from many aspects are needed for us to reach a comprehensive understanding of the swash zone hydrodynamics.

The main purpose of the present study is to provide further information on the flow characteristics in the swash zone through laboratory experiments. Unlike the previous studies, we focus on the solitary wave runup and backwash. It can be regarded as a single cycle of the swash motion induced by periodic waves and the overall phenomenon is simpler without interactions between the runup and backwash. Moreover, it is possible to carry out experiments in a larger scale, which is advantageous for the velocity measurements. The temporal variations of the bottom shear stress are estimated with different

\footnotetext{
${ }^{1}$ Department of Ocean Sciences, Tokyo University of Marine Science and Technology, 4-5-7, Minato-ku, Tokyo, 108-8477 JAPAN
} 
methods based on measured velocity profiles by LDV. Moreover, a movable bed experiment is conducted under the same condition to discuss relations of the flow properties with the sediment transport. It is expected that more clear discussions can be made on the issue due to the simplified process.

\section{EXPERIMENTAL SETUP}

\section{Facility Setup}

The laboratory experiments were carried out at Tokyo University of Marine Science and Technology in a $29 \mathrm{~m}$ long by $1.0 \mathrm{~m}$ wide wave flume. The flume consists of $1 \mathrm{~m}$ high grass walls on both sides and is equipped with a piston-type wavemaker at one end. A composite slope of 1/10 and $1 / 20$ slope was constructed at the other end of the flume as shown in Fig. 1. The $x$-axis was taken positive to the landward direction with $x=0$ at the original position of the wave paddle. The toe of the slope and the still water shoreline were respectively at $x=957 \mathrm{~cm}$ and $x=1687 \mathrm{~cm}$. The water depth in the constant depth region was set to be $44 \mathrm{~cm}$ to have the resulting swash zone within the range of the $1 / 20$ slope for a given wave condition. In the following experiments, a solitary wave with $12.0 \mathrm{~cm}$ height was generated by the wavemaker according to the method by Goring 1978. The wave height was the maximum value for a given stroke of the wavemaker and the water depth. The measured profiles of the incident wave at $x=450 \mathrm{~cm}$ of three different runs are plotted together with the theoretical profile in Fig. 2. The incident wave agrees well with the theoretical profile and appears to be highly repeatable.
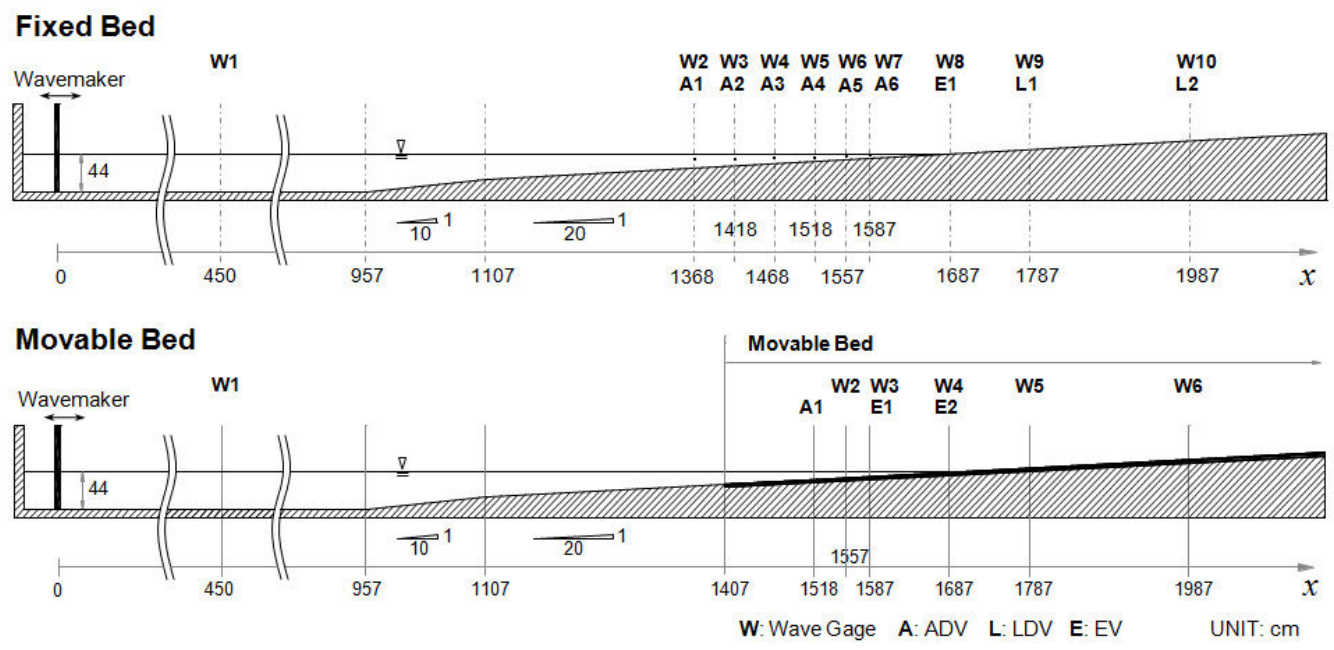

Figure 1. Wave flume setups for the fixed-bed and the movable-bed experiment.

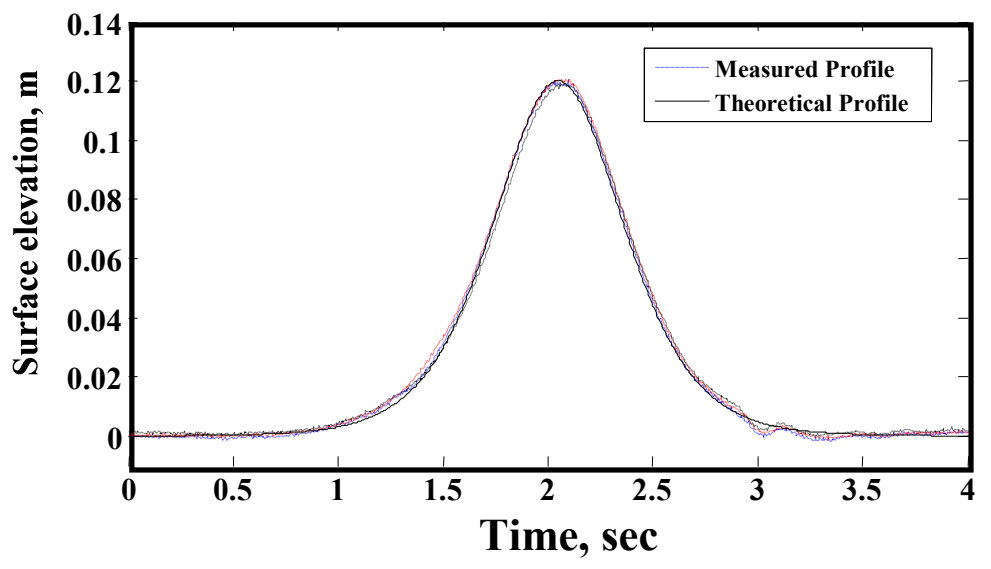

Figure 2. Measured and theoretical incident wave profiles at $x=450 \mathrm{~cm}$. 
Measurements of water surface elevations and flow velocities were conducted on five different beds. These consisted of a smooth bed, three rough beds and a movable bed as shown in Table 1. For the smooth bed, flat plates made from plastic resins (styrene acrylonitrile silicone) were rigidly fixed to the slope. The rough bed was achieved by gluing sheets of sandpaper to the upper portion of the slope from $x=1518 \mathrm{~cm}$. Three different sandpapers with $0.07,0.19$ and $0.27 \mathrm{~mm}$ diameters were used to investigate effects of different roughnesses. The experiment was also done on a movable bed consisting of silica sand with $0.15 \mathrm{~mm}$ median diameter. The movable bed was made by lowering the upper portion of $1 / 20$ slope by $5 \mathrm{~cm}$ from $x=1407 \mathrm{~cm}$ and filling the resulting space with the sand as shown in Fig. 1. Specific gravity and porosity of the sand were respectively 2.6 and 0.4 .

\begin{tabular}{|c|c|c|c|c|}
\hline \multicolumn{5}{|c|}{ Table 1. Bed conditions and properties. } \\
\hline Case & Bed Type & Bed Material & Particle Diameter, $\mathrm{mm}$ & Starting Point, $\mathrm{cm}$ \\
\hline S & Smooth & SAS &.--- & ---- \\
R1 & & & 0.07 & $\mathrm{x}=1518$ \\
R2 & Rough & Sandpaper & 0.19 & \\
R3 & & & 0.27 & $\mathrm{x}=1407$ \\
M & Movable & Silica sand & 0.15 & \\
\hline
\end{tabular}

\section{Instrumentations}

Arrangements of measurement instruments were different between the fixed and movable bed experiments as shown in Fig. 1. Water surface elevations were measured using ten capacitance-type wave gauges aligned along a centerline of the flume. The wave gauge 1 was located in the region of constant depth to measure incident wave profiles. The wave gauges 2-7 were arranged at relatively short intervals over the slope up to the still water shoreline where the incident wave goes through drastic deformations due to shoaling and breaking. For the wave gauges 8-10, a short probe with a small supporting head was used to measure small water depth over initially dry beds. The $2 \mathrm{~mm}$ thick head of the probe was installed as close as possible to the bed surface. It was found to be capable of measuring water depth larger than $3 \mathrm{~mm}$ through careful calibrations of a lower portion of the gauge

Acoustic-Doppler velocimeters with side-looking probes were installed at the same crossshore locations as the wave gauges 2-7. These velocimeters 1-6 were placed along a crossshore line $20 \mathrm{~cm}$ away from the centerline to measure the free-stream velocity synchronized with the surface elevation. The vertical positions of their measuring volumes are listed in Table 2 . Three dimensional velocity data can be obtained during the overall process at ADV 1-4 while the data acquisition was only possible until the wave runs down to the locations at ADV 5-6 due to emergence of the probe above the water surface. In order for velocity measurements in even shallower region, an electromagnetic velocimeters with L-shape probes, which are denoted as E1 and E2 in Fig. 1, were employed to obtain two dimensional velocity data. The probe was installed at $0.5 \mathrm{~cm}$ above the bed for both locations.

\begin{tabular}{|c|c|c|c|c|c|c|}
\hline \multicolumn{7}{|c|}{ Table 2. Installation height for ADV and local water depth. } \\
\hline & $\mathrm{A} 1$ & $\mathrm{~A} 2$ & $\mathrm{~A} 3$ & $\mathrm{~A} 4$ & $\mathrm{~A} 5$ & $\mathrm{~A} 6$ \\
Height from bed, $z \mathrm{~cm}$ & 6.0 & 6.0 & 4.5 & 4.5 & 2.5 & 1.0 \\
Local depth, $h \mathrm{~cm}$ & 16.0 & 13.5 & 11.0 & 8.5 & 6.5 & 5.0 \\
\hline
\end{tabular}

In the fixed-bed experiments, velocity distributions in the bottom boundary layer were measured by laser-Doppler velocimeter. The LDV system consisted of 1D fiber-optic probe, BSA signal processor (Dantec) and argon-ion laser (Spectra-Physics). The measurements were carried out at two locations landward of the still water shoreline denoted by L1 and L2 $(x=1787 \mathrm{~cm}$ and $x=1987 \mathrm{~cm})$ in Fig.1. The vertical alignments of the measuring points at the two locations are respectively illustrated in Fig. 3. The veritical profiles of horizontal velocity near the bed can be obtained by repeating the expriments with traversing the probe due to the high repeatablity of the incident wave.

In the movable-bed experiment, the bed level change due to an attack of the solitary wave was measured in two different ways. The first way was a measurement of the crossshore bed profile before and after exposed to the solitary wave. The bed profile was measured using a laser profiler along three crosshore transections. The net change in the bed level was obtained by sybstracting two profiles and taking average over three lines. The second way was to measure the time series of the instantaneous bed level throughout the cycle. This was done by taking the video of the bed evolution through the side glass wall. The sequential images were later analised to determine the bed changing rate. 

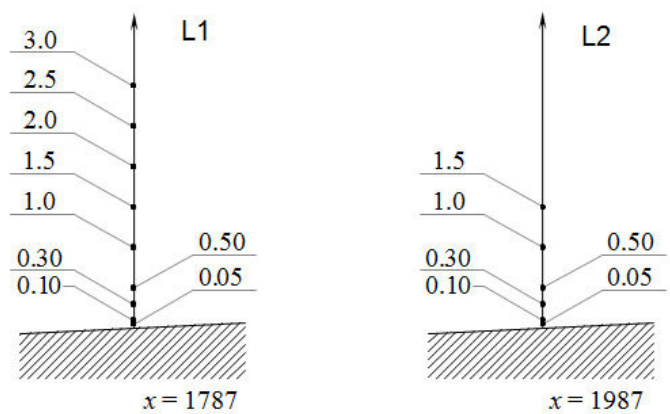

Figure 3. Vertical alignment of the measuring points by LDV at two locations.

\section{EXPERIMENTAL RESULTS}

\section{Wave Deformations over the Slope}

Fig. 4 shows the time series of water surface elevation measured along a crossshore line on the smooth bed. The first six profiles indicate the surface elevations above the still water level, while the last two profiles are taken as local water depth since the bed levels at the two locations are above the still water level. The solitary wave deformation over a slope has been already studied by many authors both theoretically and experimentally. The incident wave becomes steeper over the slope due to shoaling and results in a plunging breaker in front of the still water shoreline. The broken wave immediately evolves into a bore and then runup mode. The transition process after wave breaking will be later discussed showing series of photographs. After the runup wave reaches the highest point, the reversing flow starts to develop and finally a hydraulic jump is formed seaward of the still water shoreline as captured in profiles at $x=1557 \mathrm{~cm}$ and $1687 \mathrm{~cm}$.

Fig. 5 shows velocity profiles measured by ADV and EV at the corresponding locations to the surface elevations. At the last three locations, velocity data was not obtained for all the time due to the emergence of the probe. The wave potential energy is converted into kinetic energy through the wave breaking and thus a large velocity is created around the still water shoreline.

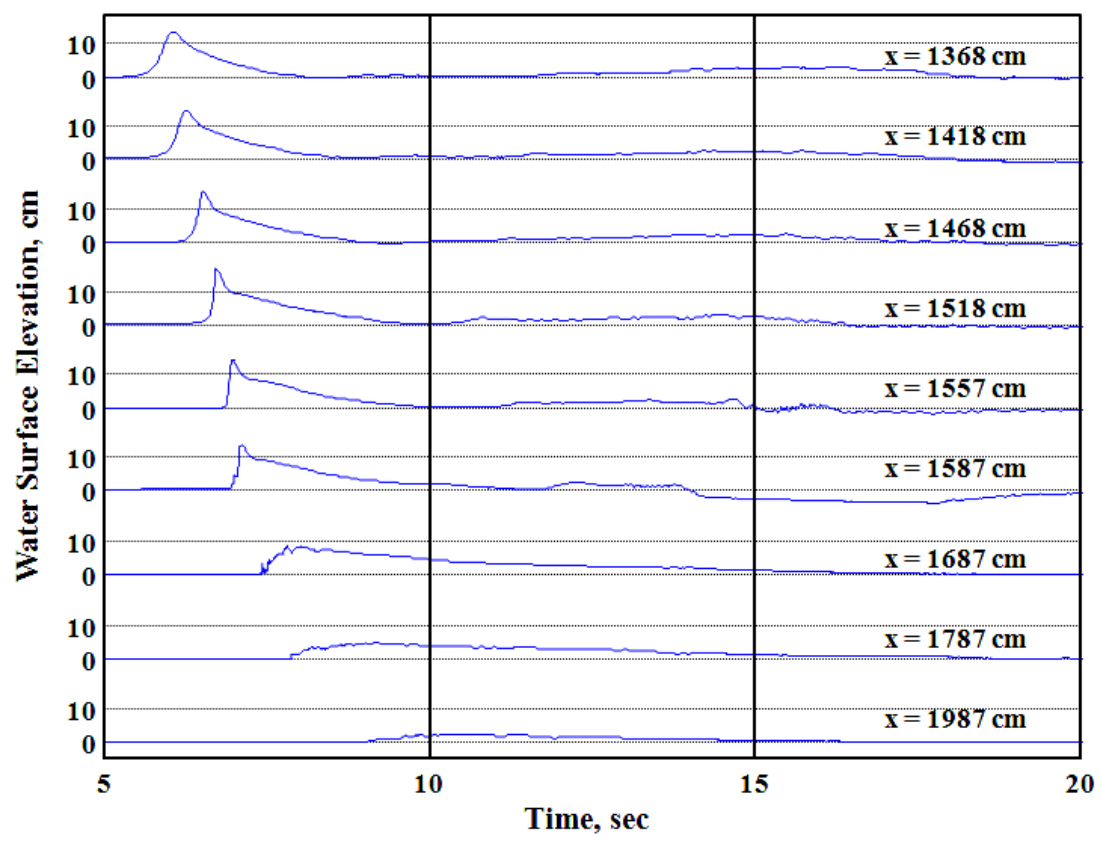

Figure 4. Measured surface profiles at wave gauges 2-9 for the smooth-bed case. 


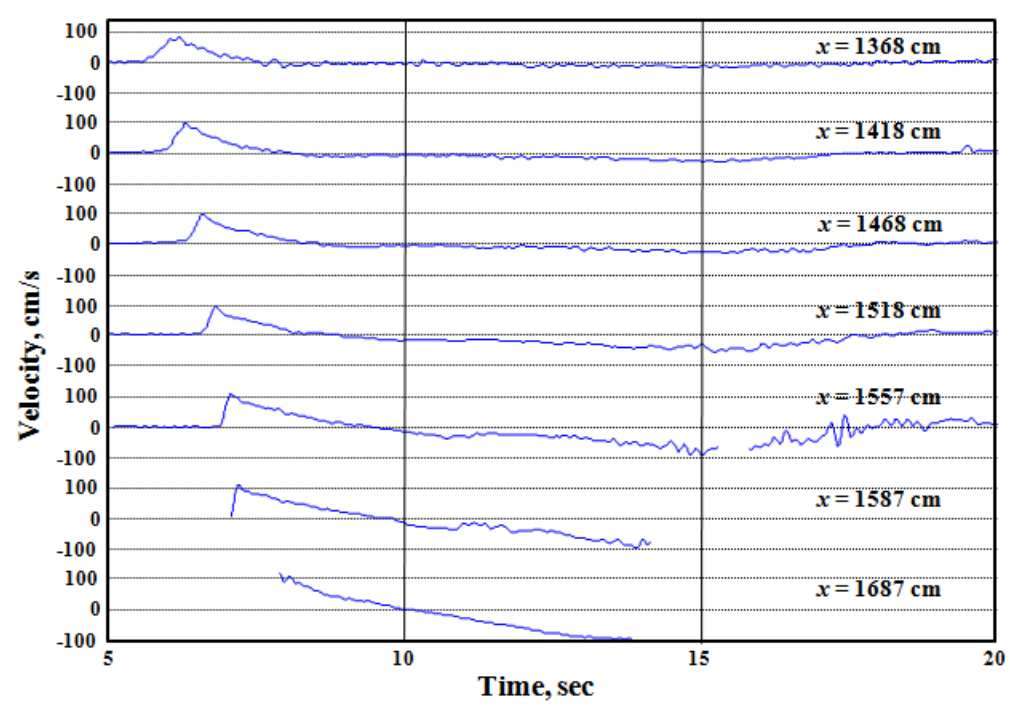

Figure 5. Measured time series of horizontal velocity at A1-A6 and E1 for the smooth-bed case.

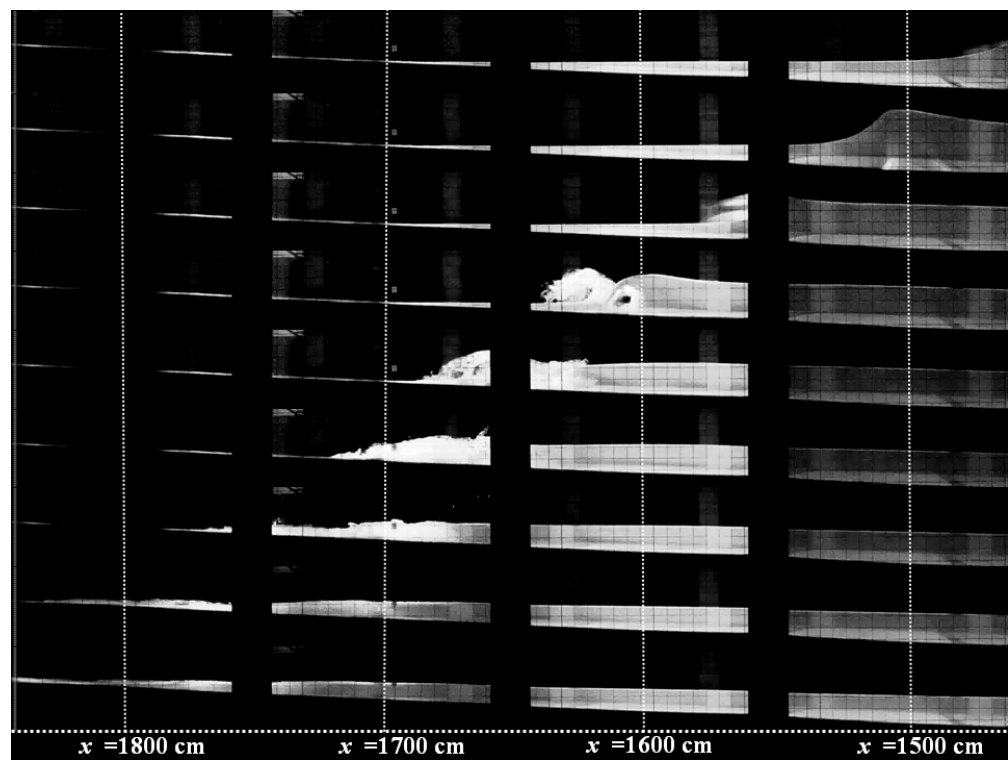

Figure 6. Transition process from wave to runup mode photographed at $0.3 \mathrm{~s}$ interval.

The wave transition into runup mode after wave breaking is illustrated in a series of snapshots at intervals of $0.3 \mathrm{~s}$ in Fig. 6. Each snapshot is composed of three lateral parts taken individually by a digital video camera to cover a wide range on the slope. This means that three parts in a snapshot are not simultaneous. Nevertheless, the series of photographs well depict the detailed process of the wave transition due to the high repeatability of the phenomenon. The plunging point of the wave breaking is located about $0.7 \mathrm{~m}$ seaward of the still water shoreline. The plunging jet pushes the quiescent water landward and produces a furious splash of water onto the foreshore. Through these processes, a considerable amount of air bubbles are entrained into water. The resulting wave becomes like a bore, but immediately transit to runup mode around the still shoreline. This behavior is known as bore collapse causing a rapid conversion of potential to kinetic energy (Yeh et al 1989). It should be noted that the process is different from that of periodic waves in which the wave energy is more gradually dissipated in the inner surfzone after wave breaking.

For later discussions, it is desirable to clarify the flow state at the measuring locations of the boundary layer flow. The first measuring location, L1 $(x=1789 \mathrm{~cm})$ is located away from the region of the drastic wave deformation. Yet turbulence and air bubbles are likely to be advected by the landward flow to the location and thus the front of the arrival wave there has a rough surface and contains a large 
amount of air bubbles. The second location, $\mathrm{L} 2(x=1987 \mathrm{~cm})$ is $3 \mathrm{~m}$ landward of the still water shoreline. Although it is out of the field of view in Fig. 6, the arrival wave was found to be much smoother and less aerated from visual observations.

\section{Waves under Different Bed Conditions}

In order to examine the influence of the different bed conditions, maximum runup heights and distances on different beds are listed in Table 3. The runup height naturally decreases as the bed becomes rougher. The result on the movable bed takes a value between R2 and R3 regardless of the smaller grain size. To see the difference in the wave, temporal variations of water depth on three different beds at the highest measuring location, $x=1987 \mathrm{~cm}$ are plotted together in Fig. 7. A total volume of water climbing up to the location significantly decreases due to an accumulative effect of the frictional resistance. The effect of the bottom roughness is significant especially at the leading and trailing edge of the wave runup. In case of the movable bed, the runup is smaller than that on fixed beds, while the water depth is larger during the backwash. The measured profile on the movable bed contains contributions of bed level changes, but it is on the order of few millimeters at this location. A possible interpretation is that infiltration-exfiltration processes play an important role. The mass and momentum flux are reduced by the infiltration process during the runup and the trapped water exfiltrated through bed sediments during the backwash.

\begin{tabular}{|c|c|c|c|c|}
\hline \multicolumn{5}{|c|}{ Table 3. Runup heights and distances under different bed conditions } \\
\hline Case & Bed Type & Particle Diameter, mm & Runup Height $R, \mathrm{~cm}$ & Runup Distance $x, \mathrm{~cm}$ \\
\hline S & Smooth & ----- & 23.4 & 2155 \\
R1 & & 0.07 & 22.4 & 2135 \\
R2 & Rough & 0.19 & 21.2 & 2110 \\
R3 & & 0.27 & 20.4 & 2095 \\
M & Movable & 0.15 & 20.9 & 2105 \\
\hline
\end{tabular}

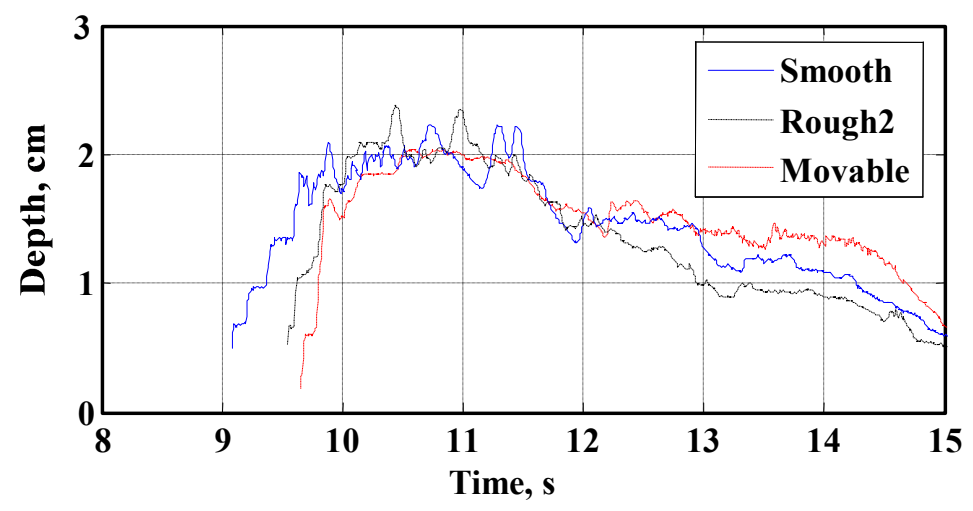

Figure 7. Comparison of the surface profiles on different beds at $x=1987 \mathrm{~cm}$.

\section{Velocity Distribution in Boundary Layer}

Fig. 8 illustrates a resulting dataset from the boundary flow measurement at $x=1787 \mathrm{~cm}$. The figure on the left shows temporal variations of water depth and horizontal velocity at four different elevations, $z=0.5,3.0,10$, and $20 \mathrm{~mm}$ on the smooth bed. Unfortunately, reliable LDV samples were not obtained in the leading tip due to the high aeration. Also in the trailing edge of the wave, water depth is too small to obtain velocity samples by LDV. The velocity measurement was successful in the fluid interior and the average sampling frequency of $200-300 \mathrm{~Hz}$ was attained in most cases. The velocity in the leading tip is expected to be much larger than the measured maximum value. The runup front velocity is roughly estimated at 1.6-1.8 m/s from the wave profiles shown in Fig. 4 assuming that it is equal to be wave front celerity. Since the flow during the backwash has a smaller velocity and lasts for a longer time, the overall profile appears to be highly asymmetric as reported by many previous authors in the periodic swash (e.g. Cowen et al. 2003).

The velocity profiles indicate the generation of turbulence during the active phase of the runup and backwash. As evidenced by a monotonic decrease in turbulence intensity upward, the most part is supposed to be bed-generated turbulence. There should be a bore-generated turbulence in the wave front, but it is not captured in the present data. In order to examine frequency characteristics, the 
spectrograms of all the profiles are shown in the right figure. They were created by applying the shorttime Fourier transform with a hamming window of $0.2 \mathrm{~s}$ to each profile in the left figure. There is no clear difference in the frequency domain between turbulence of two active phases.
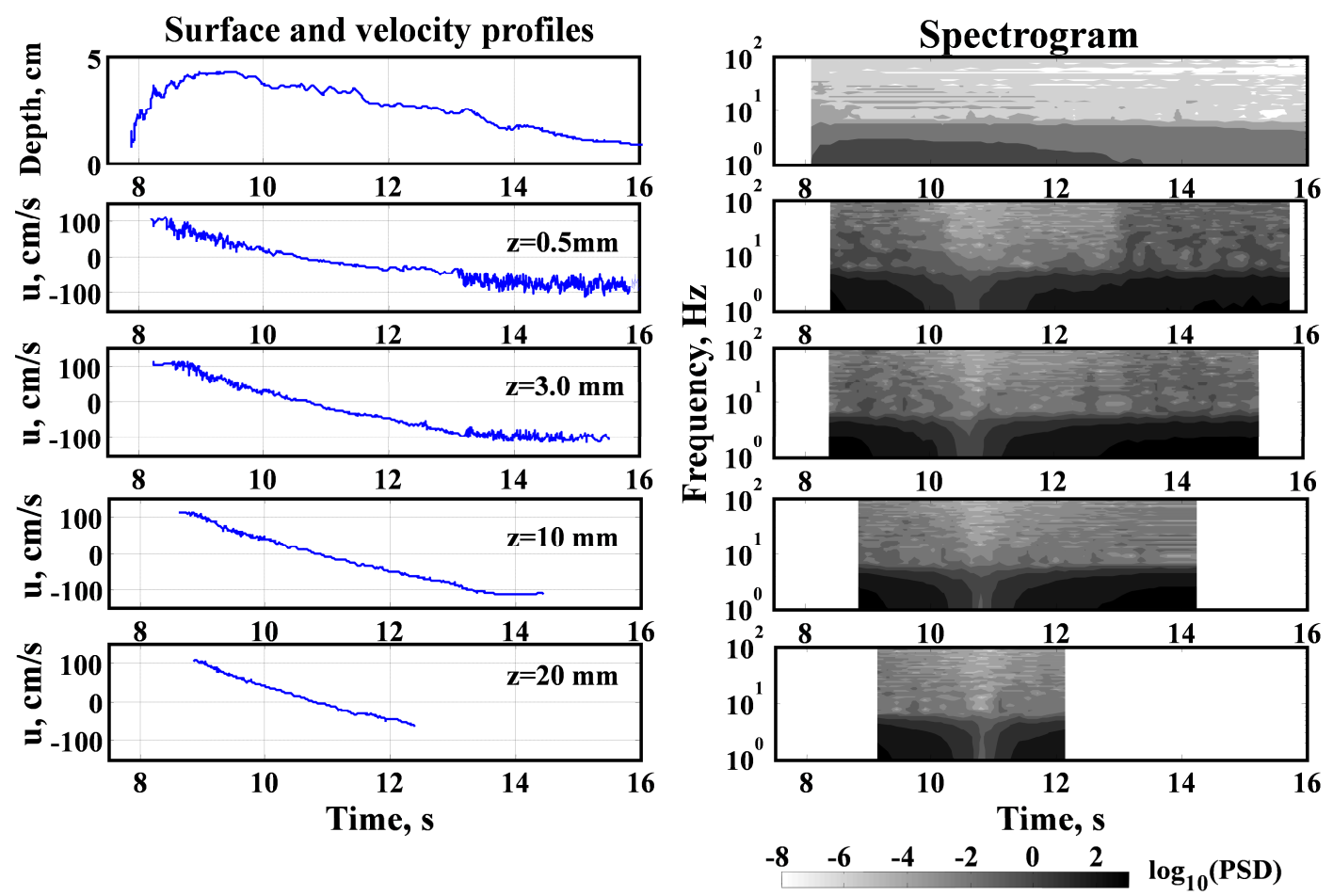

Figure 8. Measured time series of water depth and velocities at four elevations above the bed (Left panels) and their spectrograms (Right panels) at $x=1787 \mathrm{~cm}$.

In order to obtain vertical profiles of nearbed velocity, it is desirable to divide the measured velocity into a mean and a fluctuating component and then define the later component as turbulence. The phase averaging method is widely used for the velocity separation in case of the periodic wave experiment. However, it is not applicable to the present case using a solitary wave. It is not realistic to obtain a sufficient number of data for the ensemble averaging by repeating the experiment. An alternative method is a numerical filtering of the measured data in the frequency domain. A problem is a choice of cut-off frequency because both wave and turbulence components are mutually superimposed in a range of frequency domain. By checking a coherence of two different data taken under the same condition, the cut-off frequency was determined at $3 \mathrm{~Hz}$. Hence, law-frequency components below $3 \mathrm{~Hz}$ and the remaining components are respectively defined as the mean velocity and turbulence in the following analysis. There may be contaminations to some extent in the mean component due to wave-to-wave differences.

Fig. 9 shows the temporal variations of vertical profiles of the mean velocity and the turbulence intensity at the lower location, $x=1787 \mathrm{~cm}$. The turbulence intensity is evaluated by taking the rms of the turbulence component over $0.2 \mathrm{~s}$. Results on the smooth and the rough bed, R3 are shown together in these figures. Results under other rough-bed conditions, R1 and R2 are omitted as they take intermediate values between the two results above. The vertical profiles of the mean velocity and the turbulence intensity right after the wave front indicate a development of a turbulent boundary layer. The profiles around the flow reversal point show a slight phase lead in the velocity profiles near the bed. The turbulent boundary layer is newly observed during the active period of backwash. In the fluid interior, there is no significant difference in the vertical profiles between the runup and backwash. The result on rough bed is slightly deviated from that on smooth bed near the leading and trailing edge of the wave.

Fig. 10 shows the corresponding results at the upper location, $x=1987 \mathrm{~cm}$. The free-stream velocity is smaller throughout the cycle than that at L1 due to a conversion from kinetic to potential energy over the slope. Unlike the results at L1, the development of the turbulent boundary layer is only observed near the leading tip. During the active period of backwash, the turbulence intensity is not recognized 
near the bed and the velocity profiles are more likely to be of the laminar boundary layer. The asymmetric profile of the velocity leads to the difference in the flow regime between the runup and backwash at the upper location of the slope. Although it depends on an experimental scale, the result suggests that the backwash flow tends to be laminar at upper portion of the slope. Differences in water surface profiles on the smooth and the rough bed become more significant at this location, while the flow profiles agree with each other in the fluid interior. Near the leading and trailing edge, reliable data was not obtained due to the small depth on the rough bed.

In previous studies on the flow characteristic under the swash motions, it was reported that the boreinduced turbulence is dominant during the runup while the bed-generated turbulence is dominant during the backwash (Petti and Longo 2001, Cowen et al. 2003). The reported tendency is however contrary to the present results showing a decreasing profile of the turbulence intensity above the bed during both runup and backwash. A possible reason for the discrepancy is that there is no interaction between the runup and backwash in the present case with a solitary wave. The interaction may contribute to weakening of the asymmetric property and strengthening of the bore structure.
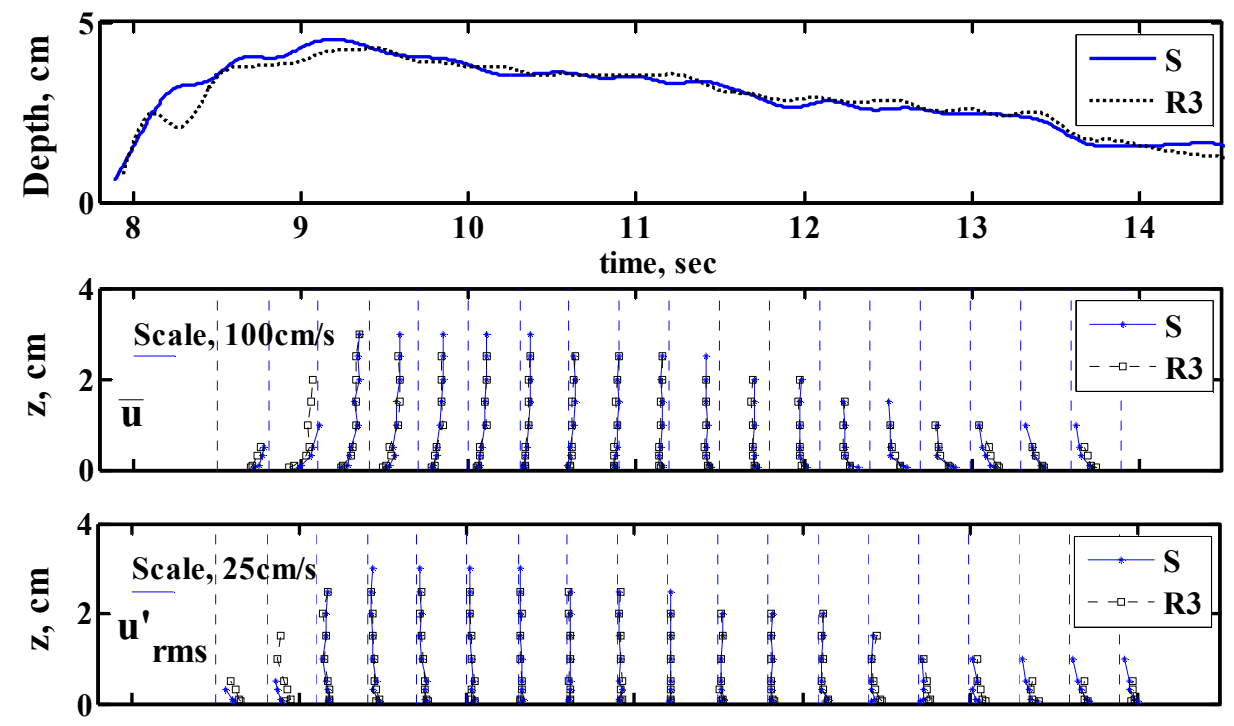

Figure 9. Temporal variation of the water depth (Upper panel), the vertical distribution of the mean velocity (Middle pannel) and the turbulence intensity (Lower panel) on two different beds at $x=1787 \mathrm{~cm}$.
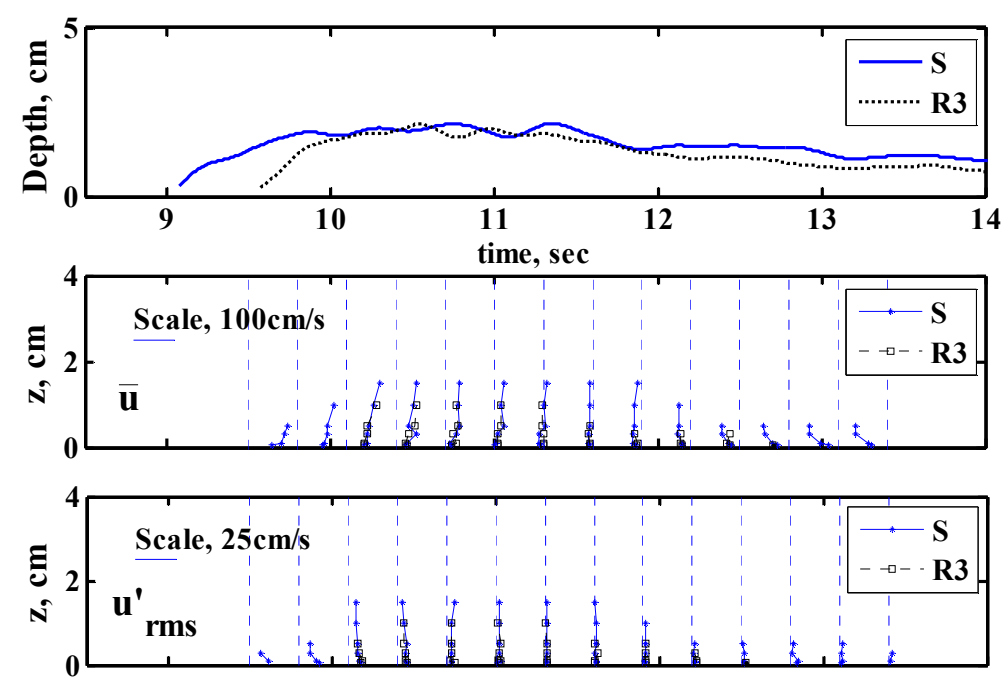

Figure 10. Temporal variation of the water depth (Upper panel), the vertical distribution of the mean velocity (Middle pannel) and the turbulence intensity (Lower panel) on two different beds at $x=1987 \mathrm{~cm}$. 


\section{Estimation of the Bottom Shear Stress}

Knowledge of the shear stress acting on the bottom is of primary importance to understand the process of sediment transport in the swash zone. The temporal variation of the bottom shear stress is estimated from the measured dataset with different methods in this section. The first way of the estimation is the least square fitting of the logarithmic profile to the measured profile following Cox et al. 2001. The method is widely used in estimations of the bed shear stress under unsteady flows despite the fact that the logarithmic profile is theoretically approved only in a fully developed turbulent boundary layer. In the following analysis, the velocity data at four elevations within $5 \mathrm{~mm}$ of the bottom are used for the velocity profile.

The above method is not expected to be reliable if the flow is laminar. The result of the present measurement actually suggests a developement of the laminar boundary layer during the backwash at the upper location of the slope. It is known that the bottom shear stress in a laminar flow is expressed as

$$
\frac{\tau_{b}}{\rho}=\left.v \frac{\partial u}{\partial z}\right|_{z=z_{b}}
$$

where $\tau_{b}$ is the bottom shear stress, $\rho$ is the fluid density, $u$ is the horizontal velocity, $z_{b}$ is the bottom elevation with respect to an arbitrary horizontal datum and $v$ is the kinetic viscosity. Hence, the bottom shear stress can be estimated from the vertical velocity gradient near the bottom. It is possible to evaluate the velocity gradient from the measured profiles by the linear least square fitting. The velocity data at three elevations within $3 \mathrm{~mm}$ of the bottom are presently used for the linear fitting. This method is expected to be capable of giving a better estimation if the boundary layer flow is laminar.

Another possible approach from the present dataset is to estimate it from the momentum balance in the horizontal direction. The bottom shear stress in shallow water is approximated by conservation of the depth-integrated momentum as

$$
\frac{\tau_{b}}{\rho}=-\frac{\partial}{\partial t} \int_{z_{b}}^{\eta} u d z-\frac{\partial}{\partial x} \int_{z_{b}}^{\eta} u^{2} d z-g D \frac{\partial \eta}{\partial x}
$$

where $g$ is the gravitational acceleration, $\eta$ is the surface elevation with respect to the horizontal datum same as $z_{b}$ and $D$ is the total depth. The first term on the right hand side accounting for the fluid acceleration is numerically evaluated from the measured velocity profile. Due to the lack of data near the surface, velocities above $z=1.0 \mathrm{~cm}$ are assumed to be vertically constant to implement the numerical integration. The assumption is supposed to be valid at least in the fluid interior as shown in Fig. 9 and Fig. 10. The surface gradient on the third term is calculated with the measured surface elevations at two neighboring locations. A remaining term to be evaluated is the second term accounting for effects of the nonlinear advection. Without data regarding the spatial difference of the horizontal velocity, it can not be directly evaluated from presently available data. The effect of the nonlinear advection is not negligible as the swash flow is highly transient. In order to overcome the problem, the second term is approximated through the continuity equation as

$$
\frac{\partial}{\partial x} \int_{z_{b}}^{\eta} u^{2} d z \approx-2 U \frac{\partial \eta}{\partial t}-U^{2} \frac{\partial D}{\partial x}
$$

where $U$ is the depth-averaged velocity to be determined from the measured velocity profiles. Now that all the terms on the right hand side of Eq. 2 are computable from the present dataset, Eq. 2 provides us an alternative way to estimate the bottom shear stress as long as the velocity at $z=1.0 \mathrm{~cm}$, is available. Unlike the other two methods based on the profile fitting, the method is valid regardless of the flow regime.

Fig. 11 shows the temporal variations of the local water depth, three terms on the right-hand side of Eq. 2 and the bottom shear stress estimated in three different ways at the two measuring locations. The estimated results are not available in the leading and trailing edge of the wave due to lack of the velocity data. Figure 11(c) and 11(d) provide us with rough sketches of the momentum balance at the two different locations on the slope. The estimated profiles of three terms have originally contained large fluctuations due to numerical differentiations of the measured data. Therefore, the time series shown in two figures are smoothed results by a $0.5 \mathrm{~s}$ moving average filter to highlight long term trends. The results suggest that the nonlinear advection plays an important role near the leading and trailing edge. It is also found that the nonlinear effect is more significant in the lower portion of the slope. 
Time series of the bottom shear stress estimated from Eq. 2 in Fig. 11(e) and 11(f), which is sum of three terms, contain long-term fluctuations corresponding to undulations in the surface elevation. The value of the shear stress is about one order of magnitude smaller than other terms in Eq. 2 and thus quite sensitive to errors in estimations of three terms. Therefore it is not clear if the fluctuations are of physical significance. In a long term trend, the result by the logarithmic fitting at $x=1787 \mathrm{~cm}$ agrees with that estimated by Eq. 2 throughout the range of data availablity. Contrary to the result at the lower location, the seaward stress at $x=1987 \mathrm{~cm}$ is considerably overestimated by the logarithmic fitting. The value is even larger than that at the lower location where the seaward velocity is much larger. This may be because the logarithmic fitting is not applicable to a laminar boundary layer. In fact, the estimations by Eq. 1 and Eq. 2 show a better agreement in this period. During the active phase of the backwash, the first and the second terms in Eq. 2 are almost canceled out so that the shear stress is balanced with the pressure gradient.

Without reliable data in the leading tip, it is not possible to discuss the landward stress from many aspects. In the result at $x=1987 \mathrm{~cm}$ in Fig. 11(f), the data is available to the vicinity of the tip region and a sharp increase in the friction is detected there. As reported by previous researchers, the maximum landward stress is marked in the leading tip of the runup wave. Therefore, there should be a narrow peak of the landward stress in the leading tip also at $x=1787 \mathrm{~cm}$ in Fig. 11(e). The magnitude of the maximum landward stress is inferred to be smaller at the upper location since the wave loses kinematic energy as it climbs up the slope.

The same analysis was carried out for the rough bed cases. However, the data availability was more severe and the result was limited only in the fluid interior. The bottom shear stress in the fluid interior does not show a significant difference from that on the smooth bed. The bottom roughness in the present study is so small that its effect is limited in the leading and trailing edge. In fact, the velocity profiles on the smooth and rough beds agree well with each other in the fluid interior as already shown in Fig. 9 and Fig. 10.
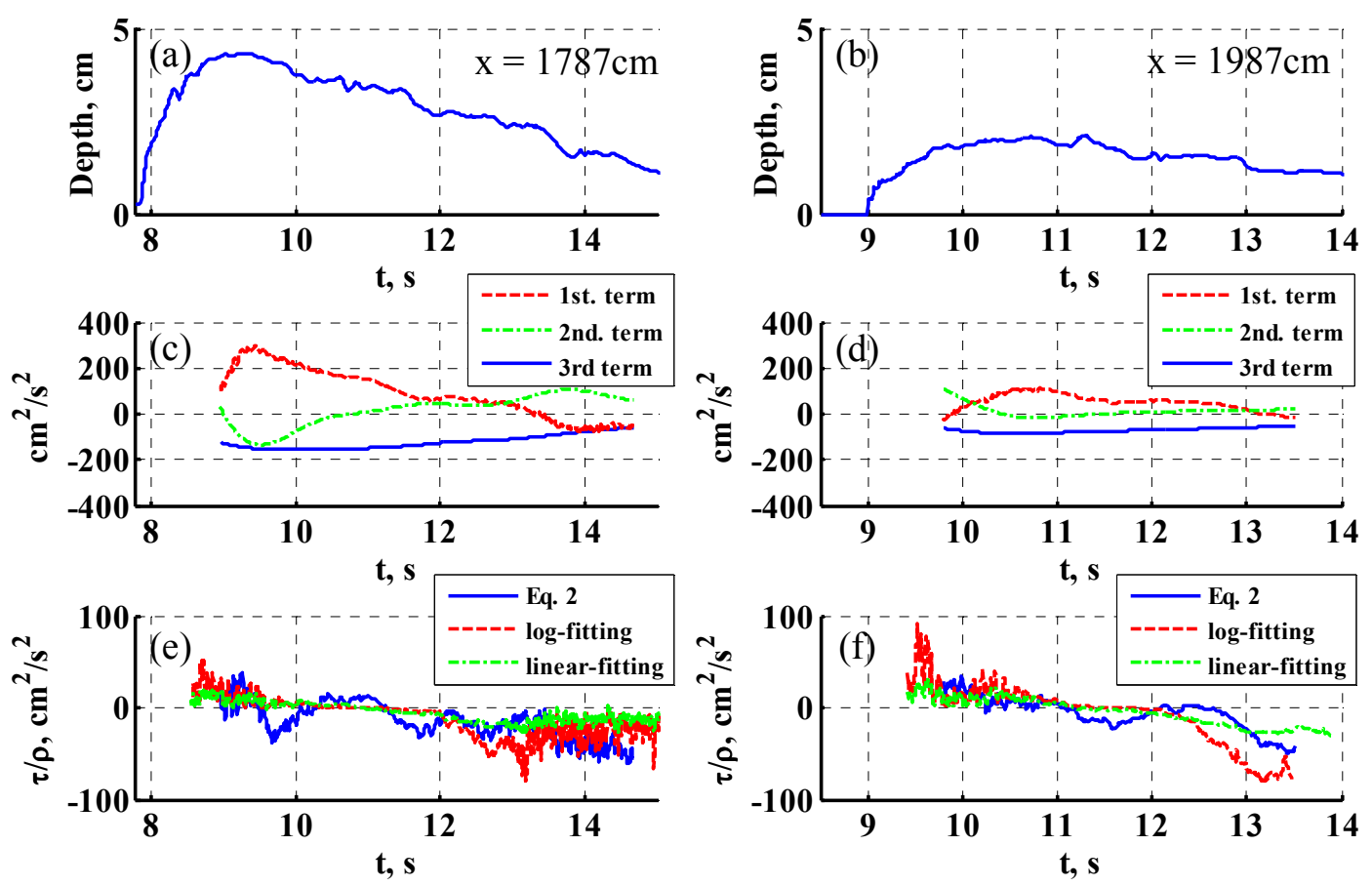

Figure 11. Temporal variations of the water depth, the three terms of the momentum equation, and the estimated shear stress at $x=1787 \mathrm{~cm}$ (Left panels) and $1987 \mathrm{~cm}$ (Light panels).

In the previous studies on the flow characteristics in swash zone, values of the friction factor is often discussed to see how much the quadratic law works for the swash flow. The main focus on the issue is variations of the friction factor over the flow phase or the location inside the swash zone. Therefore, it is worthwhile to look up the value in comparison with previous reports. The friction factor is readily calculated from the bottom shear stress as 


$$
C_{f}=\frac{2 \tau_{b}}{\rho\left|u_{0}\right| u_{0}}
$$

where $C_{f}$ is the friction factor, $u_{0}$ is the freestream velocity. $u_{0}$ is presently replaced by the velocity at $z$ $=1.0 \mathrm{~cm}$.

Fig. 12 shows the temporal variation of the friction factor at two measuring locations. The results from the logarithmic and linear fitting are plotted together in these figures. From the previous discussion on the estimated shear stress, the result by the logarithmic fitting is more reliable in Fig. 12(a). The friction factor is 0.003-0.005 during the active phases of the runup and backwash except in the leading tip where data is not available. The friction factor in the leading tip is likely to rapidly increase toward the wave front as indicated in the result at $x=1987 \mathrm{~cm}$ shown in Fig. 12(b). The sharp increase in the friction factor was also observed during the runup in the experimental result by Cowen et al. 2003. At the upper location of the slope, the friction factor significantly varies over the cycle because the flow regime is different between two active phases. The value during the active phase of the backwash estimated by the linear fitting is around 0.01 , which is larger than the value at the lower location. The friction factor during the runup shows a large variation over time indicating a sharp increase in the leading tip from $0.002-0.003$ to 0.01 . The overall tendency of the friction factor over the slope is not consistent with the experimental results by Cox et al. 2001 in which the fiction factors for both seaward and landward flows decreases over the slope from the still water shoreline. It is not easy to specify the reasons since many factors are involved in the issue.
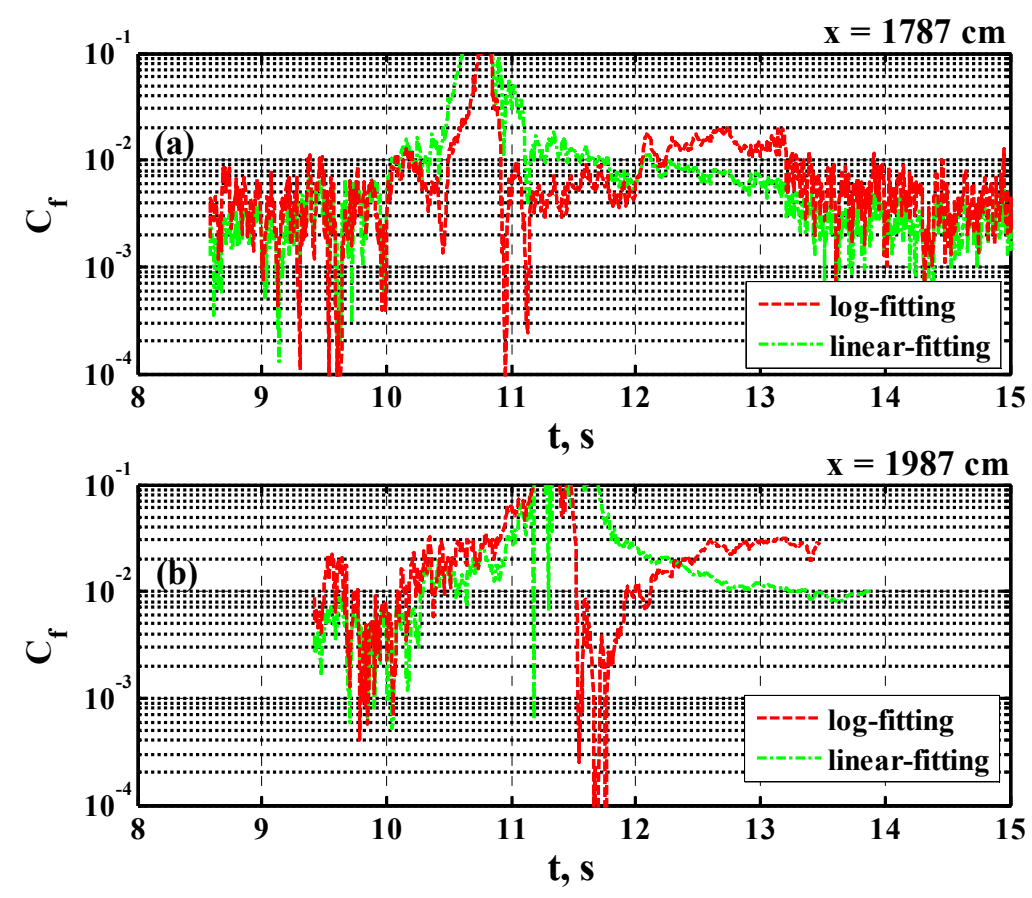

Figure 12. Temporal variations of the friction factor estimated by the logarithmic and the linear fitting at $\mathrm{x}=$ $1787 \mathrm{~cm}$ (Upper panel) and $1987 \mathrm{~cm}$ (Lower panel).

\section{Bed Level Change}

Fig. 13 shows the bed level change from the initial profile after exposed to the solitary wave. A portion of beach around the still water shoreline is heavily eroded while a large deposition is observed seaward of the still water shoreline. The profile is similar to that obtained by Kobayashi and Lawrence 2004 who investigated the sediment transport induced by a breaking solitary wave. A recent experimental study by Young et al. 2010 also focused on the same issue and they describe more detailed process and mechanism of the bed deformation. A jet induced by the furious plunging photographed in Fig. 6 entrains a considerable amount of bed sediment into water and it is transported 
landward by the subsequent flow. The runup wave climbs up while scouring the foreshore at its front and carries the sediment to the upper part of the slope, forming a small deposition there. During the backwash, the foreshore is newly eroded by the strong reversing flow and the entrained sediment is consequently accumulated through a hydraulic jump formed seaward of the still water shoreline.

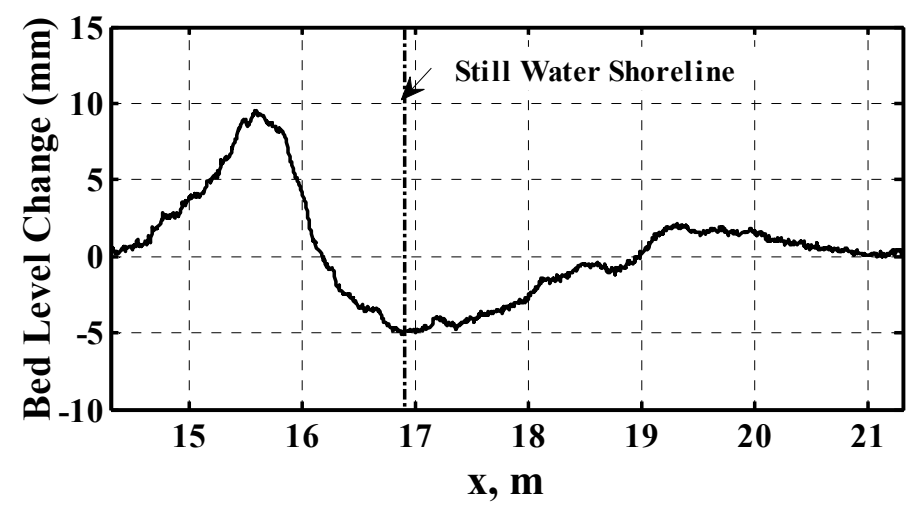

Figure 13. Bed level change induced by the solitary wave.
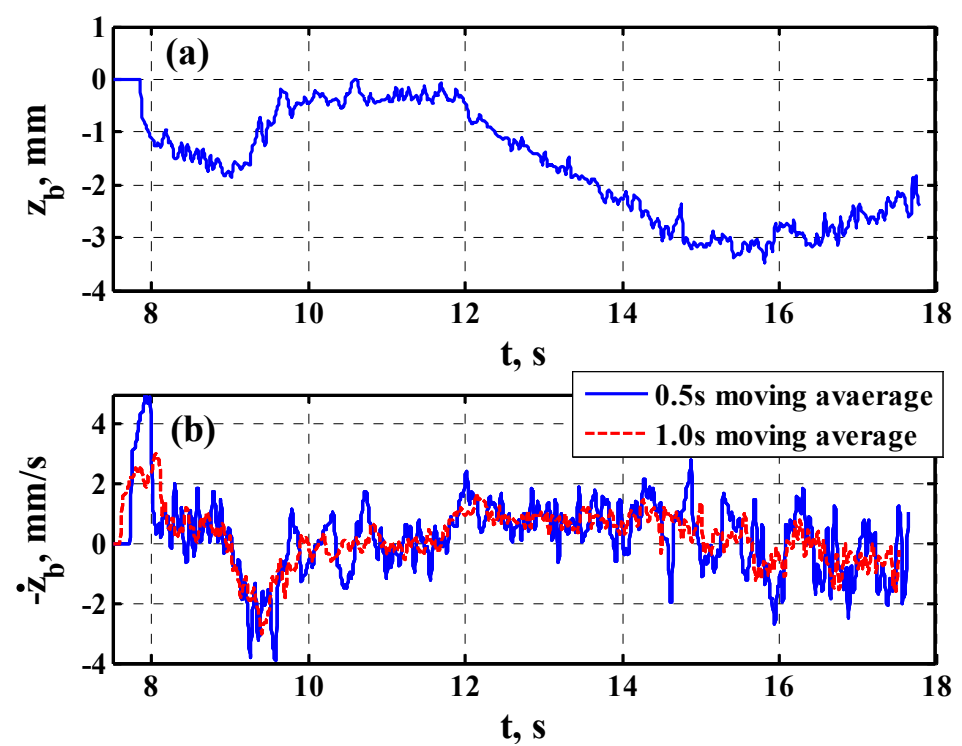

Figure 14. Temporal variations of the instantaneous bed level (Upper panel) and its changing rate (Lower panel) at $x=1787 \mathrm{~cm}$.

In order to investigate the bed evolution, a temporal variation of instantaneous bed levels is estimated from the video taken through the side glass wall at $x=1787 \mathrm{~cm}$. The instantaneous bed level is defined as a top of an unmoving part of the acquired image and determined by thresholding the frame difference. Fig. 14(a) shows the time series of the bed level change from the initial level. The bed level detection was successful except that the result contains slight fluctuations due to errors in the image process. The final bed level is slightly smaller than that at $x=1787 \mathrm{~cm}$ shown in Fig. 13 because the bed level near the side glass wall is measured in this case. It is found that the bed is rapidly scoured almost at the same time as the wave arrival. The loss of the bed sediment is mostly recovered by the landward sediment transport by the subsequent flow. As the backwash starts, the bed level again goes down with a less speed, but for a longer time than the initial scour. As a result, the bed level falls by 2.0 $\mathrm{mm}$ after the cycle.

Fig. 14(b) shows the changing rate of the bed level at $x=1787 \mathrm{~cm}$. The two curves are respectively passed through the $0.5 \mathrm{~s}$ and $1.0 \mathrm{~s}$ moving average filters to eliminate fluctuations arising from the detection errors. A decrease in the bed level is taken positive so that a larger positive value indicates more rapid erosion. In reference to Fig. 11, the result suggests a clear dependency of the bed erosion on the bottom shear stress. The large stress in the leading tip appears to significantly contribute to the net bed deformation. Although the seaward stress during the backwash is smaller in magnitude, the net 
effect is much larger due to its longer duration. It can be concluded that balances of the magnitudes and duration of the exerted shear stress during two major phases determine a direction of the bed level change. At the upper location of the slope, the initial scour is immediately tuned into a deposition during the runup and then the seaward stress is not enough to remove the deposition completely during the backwash.

\section{CONCLUSIONS}

Laboratory experiments were performed to examine the flow characteristics and its relation to the sediment transport during the runup and the backwash of the solitary wave. Flow measurements were attempted by using LDV to estimate the bottom shear stress at two locations landward of the still water shoreline. A movable bed experiment was also carried out under the same geometry to relate the bed evolution to the flow characteristics. Based on the experiments, the following conclusion can be drawn.

1. The boundary layer flow was turbulent during the active periods of both runup and backwash at the lower location, while turbulences were only admitted during runup at the higher location of the slope. The velocity data indicates the dominance of bed-generated turbulences during both phases.

2. The effect of the bottom roughness becomes significant only in the leading and trailing edge of the wave. In the fluid interior, there was no clear difference among velocity profiles on different beds. This may be because that the present range of the bottom roughness was small relative to the height of the viscous sublayer.

3. It was found from the analysis of a momentum balance that the effect of the nonlinear advection plays an important role throughout the process. The importance is relatively larger at the lower location in the swash zone. The result of the analysis also suggested that the bottom shear stress is almost balanced with the surface gradient during the active period of the backwash.

4. The bottom shear stress estimated by the logarithmic fitting roughly agrees with that resulting from the depth-integrated momentum equation if the boundary layer flow is turbulent. The method results in an overestimation when applied to a laminar flow.

5. The estimated value of the friction factor is 0.003-0.005 during both flow phases at the lower location except in the leading tip where data is not presently available. The value at the higher location shows a large variation over the cycle mainly due to the difference of the flow regime from phase to phase. The friction factor in the leading tip is inferred to be much higher than that in the fluid interior. Therefore, relevant discussions should be separately made in two flow phases during the runup.

6. The analysis of the temporal variation of the bed level revealed the detailed processes of the bed evolution by a solitary wave. The overall process can be roughly divided into three phases. The initial scour by the large stress in the wave tip is followed by the rapid deposition due to the landward sediment transport by the subsequent flow and then the erosion of long duration by the seaward flow during the backwash.

7. The result from the movable-bed experiment suggested a clear dependency of the bed deformation on the bottom shear stress. A balance of the bottom shear stress in terms of magnitude and duration between two flow phases determine a direction of local bed deformation.

\section{ACKNOWLEDGMENTS}

This research was supported by JSPS, Grant-in-Aid for Scientific Research(C), 21560534.

\section{REFERENCES}

Archetti, R. and M. Brocchini. 2002. An integral swash zone model with friction: an experimental and numerical investigation, Coastal Engineering, 45, 89-110.

Barnes, M.P., T. O’Donoghue, J.M. Alsina, and T.E. Baldock. 2007. Direct bed shear stress measurements in bore-driven swash, Coastal Engineering, 56, 853-867.

Cowen, E.A., I.M. Sou, P.L.F. Liu, and B. Raubenheimer. 2003. Particle image velocimetry measurements within a laboratory-generated swash zone, Journal of Engineering Mechanics, 129, 1119-1129.

Cox, D.T., W.A. Hobensack, and A. Sukumaran. 2001. Bottom stress in the inner surf and swash zone, Proceedings of $27^{\text {th }}$ International Conference on Coastal Engineering, ASCE, 108-119.

Goring, D.G. 1978. Tsunamis-The propagation of long waves on to a shelf, Rep. KH-R-38, W. M. Keck Lab., Cal. Inst. of Technol., Pasadena,. Cal. 
Kobayashi, N., and A. Lawrence. 2004. Cross-shore sediment transport under breaking solitary waves, Journal of Geophysical Research, 109, C030047.

Petti, M. and S. Longo, 2001, Turbulence experiments in the swash zone, Coastal Engineering, 41, 124.

Puleo, J.A., and K.T. Holland. 2001. Estimating swash zone friction coefficients on a sandy beach. Coastal Engineering, 43, 25-40.

Raubenheimer, B., S. Elgar, and R.T. Guza. 2004. Observations of swash zone velocities: A note on friction coefficients, Journal of Geophysical Research, 109, C01027.

Yeh, H. H., A. Ghazali, and I. Marton (1989). Experimental study of bore run-up, journal of fluid mechanics, 206 , 563-578

Young, Y.L., H. Xiao, and T. Maddux. 2010. Hydro- and morpho-dynamic modeling of breaking solitary waves over a fine sand beach. Part I: Experimental study, Marine Geology, 269, 107-118. 